\title{
Investigating Visual-Tactile Interactions over Time and Space in Adults with Autism
}

DOI:

10.1007/s10803-015-2492-8

\section{Document Version}

Accepted author manuscript

Link to publication record in Manchester Research Explorer

\section{Citation for published version (APA):}

Poole, D., Gowen, E., Warren, P. A., \& Poliakoff, E. (2015). Investigating Visual-Tactile Interactions over Time and Space in Adults with Autism. Journal of Autism and Developmental Disorders, 45(10), 3316-3326.

https://doi.org/10.1007/s10803-015-2492-8

\section{Published in:}

Journal of Autism and Developmental Disorders

\section{Citing this paper}

Please note that where the full-text provided on Manchester Research Explorer is the Author Accepted Manuscript or Proof version this may differ from the final Published version. If citing, it is advised that you check and use the publisher's definitive version.

\section{General rights}

Copyright and moral rights for the publications made accessible in the Research Explorer are retained by the authors and/or other copyright owners and it is a condition of accessing publications that users recognise and abide by the legal requirements associated with these rights.

\section{Takedown policy}

If you believe that this document breaches copyright please refer to the University of Manchester's Takedown Procedures [http://man.ac.uk/04Y6Bo] or contact uml.scholarlycommunications@manchester.ac.uk providing relevant details, so we can investigate your claim.

\section{OPEN ACCESS}


Investigating visual-tactile interactions over time and space in adults with autism.

\begin{abstract}
It has been suggested that the sensory symptoms which affect many people with autism spectrum conditions (ASC) may be related to alterations in multisensory processing. Typically, the likelihood of interactions between the senses increases when information is temporally and spatially coincident. We explored visual-tactile interactions in adults with ASC for the first time in two experiments using low-level stimuli. Both participants with ASC and matched neurotypical controls only produced visualtactile interactions to near simultaneous stimuli, suggesting that temporal modulation is unaffected in the adult population. We also provide preliminary evidence that visual-tactile interactions may occur over greater spatial distances in participants with ASC, which merits further exploration.
\end{abstract}

\title{
Keywords
}

Autism, visual-tactile, multisensory, selective attention, crossmodal congruency task 
Atypical sensory functioning represents a significant aspect of the experience of many individuals with autism spectrum conditions (ASC; Crane, Goddard, \& Pring, 2009; Kern et al., 2006; Leekam, Nieto, Libby, Wing, \& Gould, 2007; O'Neill \& Jones, 1997) and has recently been included as part of the diagnostic characterisation of the condition (American Psychiatric Association, 2013). Common categories of sensory symptom include sensory over-responsiveness (e.g. sensitivity to fluorescent lighting or light touch), sensory under-responsiveness (e.g. apparent raised threshold to pain or temperature) and sensory seeking behaviours (e.g. fascination with lights or excessive smelling of objects; see Hazen, Stornelli, O'Rourke, Koesterer, \& McDougle, 2014 for a recent review of sensory symptoms). The nature of these sensory symptoms varies between individuals and the aetiology remains unknown. It has been proposed that sensory symptoms may arise from a disruption in the interaction between the senses in ASC (larocci \& McDonald, 2006). Effective multisensory interactions are integral in creating a coherent perception of our environment; it is important that only information originating from the same source interacts. Efficient processing of multisensory stimuli develops throughout childhood (Gori, Del Viva, Sandini, \& Burr, 2008; Hillock-Dunn \& Wallace, 2012). It is possible that abnormalities in neuronal development in ASC could disrupt the maturation of these processes, leading to altered multisensory processing. For instance, reduced long range connectivity in ASC which may lead to issues integrating information between networks (Anderson et al., 2011; Brock, Brown, Boucher, \& Rippon, 2002; Kikuchi et al., 2014).

There are a number of studies investigating multisensory interactions in ASC using audio-visual speech stimuli in the McGurk task (MacDonald \& McGurk, 1978). When presented with speech sounds that do not match lip movements, participants with ASC report that they perceive the sound, rather than the fused multisensory percept. This could indicate general difficulties with multisensory processing (Smith \& Bennetto, 2007), but could also be driven by problems with lip reading (larocci, Rombough, Yager, Weeks, \& Chua, 2010; Taylor, Isaac, \& Milne, 2010; Williams, Massaro, Peel, Bosseler, \& Suddendorf, 2004). Importantly, the social and semantic stimuli used in this paradigm increases the difficulty of the task for individuals with autism, independently of the multisensory demands. Tasks utilising low-level stimuli allow differences in multisensory processing to be explored, circumventing the difficulties that social and semantic stimuli can present to individuals with ASC. 
A large body of research has characterised multisensory interactions in neurotypical (NT) populations using simple low-level stimuli. Typically these tasks involve participants responding to stimuli presented in one modality which may then be influenced when stimuli from a different modality are introduced. Two such tasks are the flash-beep illusion (Shams, Kamitani, \& Shimojo, 2000) and the crossmodal congruency task (CCT; Spence, Pavani, \& Driver, 1998). The flash- beep illusion is an audio-visual task where participants perceive multiple (illusionary) flashes when presented with multiple beeps (Shams et al., 2000; Shams, Kamitani, \& Shimojo, 2002). Report of the illusion is used as a measure of audio-visual interactions. The CCT is a measure of the interaction between vision and touch; participants typically judge the elevation of vibrotactile stimuli presented at the top and bottom of a foam cube while presented with near simultaneous visual distractors. Participants are faster and more accurate when these distractors are congruent with the target vibration (flash and vibration at the same elevation). Conversely, reaction times are delayed, and error rate increased for incongruent stimuli (flash and vibration at different elevations). The congruency effect (CE) is the difference in performance between congruent and incongruent trials and is used as a measure of visual-tactile interactions. A number of studies have indicated that these multisensory interactions measured using the CCT and flash-beep illusion increase when stimuli occur close together in time and space (e.g. Shams et al., 2002; Shore, Barnes, \& Spence, 2006; Spence, Pavani, \& Driver, 2004). That is, multisensory interactions are temporally and spatially modulated.

There is evidence that low-level multisensory processing is typical in adults with ASC. The illusory flash-beep percept has been reported on a similar proportion of trials by participants with ASC and matched NT controls (Keane, Rosenthal, Chun, \& Shams, 2010; van der Smagt, van Engeland, \& Kemner, 2007). Adults with ASC and NT have also produced a similar performance enhancement on a visual search task accompanied by task-irrelevant auditory information (de Boer-Schellekens, Keetels, Eussen, \& Vroomen, 2013; although see Collignon et al., 2013). However, a recent study has indicated that the illusory flash-beep percept is experienced less frequently in children with ASC than NT controls (Stevenson et al., 2014). Furthermore, children with ASC do not show typical performance enhancements when presented with multisensory stimuli over what would be expected if the stimuli were processed independently (Brandwein et al., 2013). 
Further differences have been reported in participants with ASC relative to NT when the temporal presentation of audio-visual stimuli has been manipulated. Children with ASC exhibit multisensory effects over a range of stimulus onset asynchronies (SOAs) which is twice the size of that seen in NT children (Foss-Feig et al., 2010; Kwakye, Foss-Feig, Cascio, Stone, \& Wallace, 2011). Evidence of multisensory interactions have been reported up to around $\pm 150 \mathrm{~ms}$ SOA in NT children and $\pm 300 \mathrm{~ms}$ for children with ASC (where negative SOA indicates that beeps preceded the visual target). This suggests that the temporal modulation of audio-visual information may be altered in ASC. However, a study of adolescents and young adults using a number of audio-visual tasks indicated that temporal modulation was statistically indistinguishable from controls (de Boer-Schellekens et al., 2013). In summary, low level audio-visual interactions appear to be altered in children with ASC, but similar when ASC and NT adults are compared. The current study is the first to explore the spatial modulation of multisensory stimuli in ASC, in addition to temporal modulation.

The following experiments are also the first to investigate low level visual-tactile interactions in ASC. A recent study examining tactile temporal order judgements between the hands has indicated that children with ASC do not produce the typical performance detriment when their hands are crossed (Wada et al., 2014). Although this is a purely tactile task, the hands crossed effect has been attributed to the visual frame of reference affecting tactile perception, which suggests that this multisensory effect may be atypical in ASC. It is important to investigate visual-tactile processing as abnormalities may contribute to an aversion to being touched in ASC (see O'Neil and Jones, 1997), and could have implications for the alterations in motor control seen in ASC (Gowen \& Hamilton, 2013). Visual-tactile interactions can provide a proxy measure of how near body (peripersonal) space is represented; being more likely to occur when visual stimuli are presented within the peripersonal space surrounding the hand (see Spence, Pavani, Maravita, \& Holmes, 2004). Peripersonal space plays a subtle, but important role in social interaction. Typically we do not allow strangers to encroach our peripersonal space, and it can be disturbing if they do so. The representation of peripersonal space in ASC may affect higher level social behaviour, for instance, in maintaining socially appropriate distances (see Lloyd, 2009).

We explored two aspects of visual-tactile processing in a sample of adult participants with ASC and matched NT controls. Firstly, we aimed to determine whether the temporal modulation of visual-tactile 
interactions is disrupted in participants with ASC, as has previously been reported for audio-visual interactions (Foss-Feig et al., 2010; Kwakye et al., 2011; Stevenson et al., 2014). Secondly, we sought to provide novel insight into the spatial modulation of multisensory interactions in ASC. In order to do so, we used an adapted version of the CCT (Spence et al.,1998), somewhat analogous to the flash-beep illusion (Poole, Couth, Gowen, Warren, \& Poliakoff, 2015). Participants were asked to judge whether they felt a single or double tactile pulse while presented with distracting light flashes (Holmes, Sanabria, Calvert, \& Spence, 2006). Distractors were congruent (e.g. one pulse, one flash) or incongruent (one pulse, two flashes). As with the typical version of the task, the difference between the congruent and incongruent conditions gives the CE. In the present version of the task, unisensory (tactile) performance was measured and thresholded so that performance across participants was fixed at around $75 \%$ accuracy. There were a number of reasons for adopting this technique: firstly, any confounds relating to differences in tactile performance that may exist between the groups were removed (Blakemore et al., 2006; Puts, Wodka, Tommerdahl, Mostofsky, \& Edden, 2014).

Furthermore, tactile thresholds have previously been found to positively correlate with autistic traits in NT participants (Poole et al., 2015), and presenting stimuli at each participant's threshold should also reduce variability in tactile performance within the control group. Secondly, this enabled the CE to be measured through error rates rather than reaction times. Reaction time data can be variable in participants with ASC (Geurts et al., 2008) and this could potentially mask any differences in multisensory processing between the groups. Finally, comparing performance in conditions including visual distractors to unisensory performance provides an additional measure of multisensory interactions, specifically facilitation effects driven by congruent distractors can be separated from interference effects in response to incongruent distractors.

\section{Method}

\section{Participants}

18 participants with ASC (2 female) plus 18 NT controls matched for age, sex, handedness and IQ took part in the study. A previous study reported a significant effect of temporal and spatial modulation of visual-tactile interactions in 13 NT participants (Poole et al., 2015). In the present study we aimed to exceed this sample size to find a between groups effect and account for the heterogeneity likely in participants with ASC. Participants were recruited through adverts in the local community, the Autistic 
Society Greater Manchester and Tameside Autism Network. All ASC participants had previously received a diagnosis through clinical assessment (DSM IV criteria, APA, or ICD-10). Diagnosis was confirmed using module 4 of the Autism Diagnostic Observation Schedule (ADOS-2; Lord, Rutter, DiLavore, \& Risi, 2012) by a certified assessor. ADOS severity scores ranged from $5-12: n=6$ reached the criteria for autism, $n=11$ for autism spectrum and $n=1$ did not reach the cut-off for an ASC $^{1}$. Autistic traits were measured in our NT sample using the Autism Spectrum Quotient (AQ; Baron-Cohen, Wheelwright, Skinner, Martin, \& Clubley, 2001). AQ responses ranged from 9 - 28. No participants exceeded the cut-off of 32 which is believed to indicate clinical significance. All participants had a full scale IQ $>80$ measured using the Wechsler Abbreviated Scale of Intelligence (WASI, Weschler, 1999) and were aged 19-42 (see Table 1 for participant characteristics). Four participants with ASC and four controls were reported as left handed using the Edinburgh Handedness Inventory (EHI, Oldfield, 1971). All participants had normal or corrected to normal vision (6/6 vision in both eyes as measured using Snellens test of visual acuity), and 60s arc stereo acuity as measured using the TNO test. No participant reported a history of a visual disorder. All participants gave written consent and the study was approved by the University of Manchester ethics committee in accordance with the Declaration of Helsinki.

One participant with ASC (female, right handed) was excluded from Experiment 2 prior to analysis due to responding randomly through fatigue (baseline accuracy rate $=40 \%$ ), and was unavailable to return for a second session.

$<$ Table 1 goes here $>$

\section{Apparatus}

Participants sat at a desk in a dimly lit room and were instructed to focus on a central fixation point, consisting of a white cross $(19 \mathrm{~mm})$ on a computer monitor, displayed approximately $30^{\circ}$ below eye level and approximately $45 \mathrm{~cm}$ from the participant. A mirror was angled above the participant that allowed the experimenter to ensure that central fixation was maintained.

\footnotetext{
${ }^{1}$ The pattern and statistical significance of results was unaffected by removal of the participant that did not reach the ADOS cut-off. This participant is included in the analysis presented here.
} 


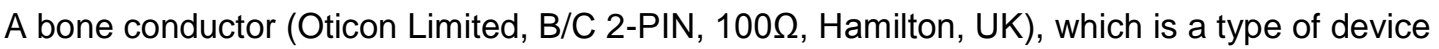
commonly used as a hearing aid, was used to present tactile stimuli (vibrations) to the participant's finger. The bone conductor was embedded within a $70 \mathrm{~mm}$ foam cube that participants held between the thumb and forefinger of their dominant hand. The cube was positioned $140 \mathrm{~mm}$ from the midline, approximately $90^{\circ}$ degrees below eye level. The participant's index finger was attached to the bone conductor using double-sided adhesive tape. The bone conductor was driven by sound files (white noise, $\sim 66 \mathrm{~dB}$ SPL) from a PC through a Tacamp amplifier (Dancer Design, St. Helens, UK) to create the tactile vibration. Six $10 \mathrm{~mm}$ red LEDs, were affixed in symmetrical locations around the monitor. The LEDs were visible through $10 \mathrm{~mm}$ holes in a black cardboard shield which surrounded the monitor. This enabled the presentation of distractors from multiple locations, while controlling for position in 3D space. Each LED was $29 \mathrm{~cm}$ from the fixation cross: one LED positioned above the bone conductor, one LED $21 \mathrm{~cm}$ above the conductor offset at an angle of $10^{\circ}$, and one LED $42 \mathrm{~cm}$ directly above the bone conductor. Three LED's were fixed in symmetrical positions on the opposite side of the monitor, allowing identical positioning for left handed participants (Fig 1).

White noise ( 75dB SPL) was played through headphones throughout the experiment to prevent the participant from hearing sounds emitted by the bone conductor. The participant's foot rested with the heel and toe each depressing a foot pedal, throughout the experiment. Responses were recorded when the participant lifted the heel or toe.

$<$ Figure 1 goes here $>$

\section{Procedure}

To confirm sensory symptoms in our ASC sample all participants completed the Adolescent/ Adult Sensory Profile (AASP; Brown \& Dunn, 2002). The AASP is a self- report Likert style questionnaire measuring trait sensory processing. Items are divided into four quadrants (low registration, sensation seeking, sensory sensitivity and sensation avoiding), across categories of sensory processing e.g. touch processing (see Table 1).

Threshold procedure 
To fix unisensory tactile performance at around 75\% accuracy experimental stimuli were delivered at approximate threshold level determined prior to the experimental procedure. Participants received two successive tactile stimuli in a two interval forced choice procedure using a constant (single) vibration and two separate (double) $80 \mathrm{~ms}$ vibrations separated by a $0-200 \mathrm{~ms}$ period of silence. The participant was instructed to indicate which interval contained the double vibration. An adaptive staircase procedure (PEST; see Taylor \& Creelman, 1967) was used to determine the duration required between two vibrations for the participant to correctly identify a double pulse $75 \%$ of the time. Further details are provided in supplementary materials.

\section{Experimental Procedure}

Participants were asked to make speeded responses upon the presentation of a target vibration, responding by lifting their toe in response to single pulses, and heel in response to double. Throughout the experimental procedure, vibrations were presented at threshold level for that participant. The task included both baseline trials and trials with task irrelevant distracting LED light flashes, which were either congruent (e.g. single vibration, single light flash) or incongruent (e.g. single vibration, double light flash). Participants were explicitly instructed to ignore the light flashes as much as possible.

The central white fixation cross remained onscreen throughout the session, however, the start of each trial was indicated by a larger, silver version of the cross $(24 \mathrm{~mm})$ appearing for $750 \mathrm{~ms}$. After a further $750 \mathrm{~ms}$ the target stimulus was delivered. Distracting light flashes were presented at SOAs according to the conditions described below. For single flashes the LED was stimulated for $80 \mathrm{~ms}$, double flashes comprised two single $80 \mathrm{~ms}$ flashes separated by $120 \mathrm{~ms}$. If the participant responded before the presentation of the vibration, the trial was discounted and the error message 'Too early' was presented for $1000 \mathrm{~ms}$. There was an inter-trial interval of $1500 \mathrm{~ms}$ following the participant's response.

Distractor and baseline unimodal trials were intermixed. In Experiment 1, the distractor lights were always presented from the $0 \mathrm{~cm}$ position. There were 9 conditions: distractors (congruent, incongruent) $x$ SOA (-30ms, $100 \mathrm{~ms}, 200 \mathrm{~ms}$ and $400 \mathrm{~ms})$, plus baseline. In Experiment 2 the distractor lights were always presented at $-30 \mathrm{~ms}$ SOA. There were 9 conditions: distractors (congruent, incongruent) $x$ position $(0 \mathrm{~cm}, 21 \mathrm{~cm}, 42 \mathrm{~cm}$, and $42 \mathrm{~cm}$ on the opposite side, referred to herein as 
$\left.42 \mathrm{~cm} \_o p p\right)$ plus baseline trials. Both experiments consisted of four experimental blocks of 90 trials, in which each condition was presented 10 times in a randomised order, with half of these trials being single vibrations and half double. This gave a total of 360 trials, with 40 trials in each condition. An enforced break was included after each block. Participants completed the AASP during a substantial break between the experiments. The order of testing was counterbalanced within the groups to avoid either experiment being unduly affected by fatigue or practice effects.

\section{Practice procedure}

Before the experimental procedure began, there were three practice blocks to familiarise participants with the task and ensure that the threshold procedure had worked effectively. The protocol for the practice procedure is provided in supplementary materials.

\section{Visual task}

At the end of the experiment there was a short visual task to ensure all participants could detect single and double light flashes at each location. There was no difference in performance between the groups, see supplementary materials for more details.

The entire experiment lasted approximately two hours.

\section{Data analysis}

Performance accuracy was calculated for each participant in each condition. Trials longer than 2000ms (Experiment 1; ASC (3.05\% of trials), NT (0.01\%). Experiment 2; ASC (1.67\%), NT $(0.37 \%)$ ), or under 150ms (Experiment 1: ASC (1.33\%), NT (0.24\%). Experiment 2; ASC (1.33\%), NT (0.17\%)) were removed from analysis. These errors were likely caused by lapses in attention or foot pedal errors and were not included in the error rate (Holmes, Sanabria, Calvert, \& Spence, 2007; Shore et al., 2006). Data point outliers \pm 2 SD from the group mean of that condition were replaced with the maximum or minimum value $\pm 2 \%$. Error rates were used to calculate the $\mathrm{CE}$, which is given by deducting the errors in incongruent from congruent trials. In Experiment 2 a square root transformation was used to correct positive skew in the CE data (Shaprio - Wilks $p>.023$ ). Mixed ANOVAs were used to compare the CE between the groups and at each SOA (Experiment 1) or 
position (Experiment 2). Significant effects and interactions were followed up with paired sample ttests. Multisensory performance was also contrasted with unisensory performance, using paired sample t-tests to compare accuracy in each condition with the baseline condition of that experiment (Fig 3). In an additional analysis Pearson's correlation coefficients were calculated to investigate the relationship between NT participant's responses to items on the AASP and AQ. The relationship between $A Q$ scores, tactile thresholds and the size of the CE in each SOA and position were also explored using Pearson's correlation coefficients. Data point outliers \pm 2 SD from the mean were replaced with the minimum/ maximum value \pm 2 .

\section{Results}

\section{Temporal Separation Thresholds}

Tactile temporal separation thresholds revealed no significant group differences for the temporal or spatial tasks (Table 1).

\section{Experiment 1: Temporal Manipulation}

The CE was larger for distractors presented at -30ms than at later SOA (Fig 2). The Group x SOA interaction was the critical test to determine whether the temporal modulation of visual-tactile interactions differed between the groups, but this interaction was not statistically significant.

A mixed ANOVA [SOA (-30ms, 100ms, 200ms, 400ms) x Group (ASC, NT)] revealed a significant effect of SOA $\left(F(3,102)=18.14, p<.001, \eta_{p}{ }^{2}=.348\right)$ indicating that the size of the CE varied across SOA. To follow up the significant effect of SOA data was pooled between the groups and paired sample t-tests (Bonferroni corrected, $\alpha=.008$ ) were conducted to compare the congruency effect between SOAs. The CE was significantly larger at $-30 \mathrm{~ms}$ than at $100 \mathrm{~ms}$ ( $\mathrm{t}(35)=3.78, p=.001, d=$ $0.68)$, 200ms (t (35) $=5.68, p<.001, d=1.24)$ and 400ms (t $(35)=5.27, p<.001, d=1.38)$. The congruency effect was significantly larger at $100 \mathrm{~ms}$ than at $200 \mathrm{~ms}(\mathrm{t}(35)=3.00, p=.005 . d=0.64$ ) and 400ms (t $(35)=3.15, p=.003, d=0.78)$, but did not differ between $200 \mathrm{~ms}$ and $400 \mathrm{~ms}(\mathrm{t}(35)$ $=.49, p=.622, d=0.12)$. The interaction between Group and SOA, was not significant $(F(3,102)=$ $\left..88, p=.453, \eta_{\mathrm{p}}^{2}=.025\right)$ indicating that the congruency effect across the SOA was similar between the groups. 
Paired sample t-tests (Bonferroni corrected, $\alpha=.006$ ) were conducted to compare performance of congruent and incongruent distractors with baseline performance (Fig 3). The effects of the distractors were similar for each group with (near) simultaneous incongruent distractors leading to an increased error rate. Participants with ASC produced an error rate significantly greater than baseline to incongruent distractors presented at $-30 \mathrm{~ms}(\mathrm{t}(17)=3.19, p<.005, d=0.92)$. There was a trend towards congruent distractors presented at 200ms $(\mathrm{t}(17)=2.33, p=.033, d=0.80)$ and incongruent distractors presented at $400 \mathrm{~ms}(\mathrm{t}(17)=2.75, p=.014, d=0.41)$ producing a lower error rate than baseline. No other differences reached statistical significance $(\mathrm{t}<2.04, p<.058)$. NT participants produced an error rate significantly greater than baseline to incongruent distractors presented at $30 \mathrm{~ms}(\mathrm{t}(17)=5.22, p<.001, d=1.76)$ and $100 \mathrm{~ms}(\mathrm{t}(17)=3.17, p=.006, d=0.88)$. No other differences reached statistical significance $(t<1.06, p>.304)$.

\section{Experiment 2: Spatial Manipulation}

The CE was marginally larger for the distractors presented by the participants hand than at more distant positions (Fig 2). The Group x Position interaction was the critical test of differences in spatial modulation of visual-tactile interactions between the groups. There was a trend towards a between group differences in the size of the CE across the positions, but this was not statistically significant.

A mixed ANOVA [Position (0cm, 21cm, 42cm, 42cm_opp) x Group (ASC, NT)] revealed a significant effect of Position $\left(F(3,99)=2.66, p=.052, \eta_{p}{ }^{2}=.075\right)$ indicating that the size of the congruency effect varied across the different positions. Paired sample t-tests (Bonferroni correction, $\alpha=.008$ ) revealed that there was a trend towards a larger congruency effect to distractors presented at $0 \mathrm{~cm}$ over $42 \mathrm{~cm}$ (t (35) $=2.58, p=.014, d=0.37)$ and $21 \mathrm{~cm}$ over $42 \mathrm{~cm}(\mathrm{t}(35)=2.17, p=.037, d=0.26)$. No other differences reached statistical significance $(\mathrm{t}<1.11, p>.276)$. The interaction between Position and Group was approaching significance $\left(F(3,99)=2.51, p=.063, \eta_{p}^{2}=.071\right)$ suggesting that the effect of position on CE tended to differ between participants with ASC and NT.

Paired sample t-tests (Bonferroni corrected, $\alpha=.006$ ) were conducted to compare performance in each condition with baseline performance (Fig 3). Incongruent distractors positioned close to the stimulated hand lead to an increased error rate for both groups, but participants with ASC also produced this effect to the distractor in the opposite hemispace. Participants with ASC produced an 
error rate significantly greater than baseline to incongruent distractors presented at $0 \mathrm{~cm}(\mathrm{t}(16)=5.78$, $p<.001, d=1.34), 21 \mathrm{~cm}(\mathrm{t}(16)=4.57, p<.001, d=1.14)$ and 42cm_opp $(\mathrm{t}(16)=3.42, p=.003, d=$ 0.98). The increase in error rate in response to the $42 \mathrm{~cm}$ light was approaching significance $(\mathrm{t}(16)=$ 2.97, $p=.009, d=0.83)$. No other differences reached statistical significance $(\mathrm{t}<1.37, p>.189)$. NT participants produced an error rate significantly greater than baseline to incongruent distractors presented at $0 \mathrm{~cm} \mathrm{(t}(17)=3.43, p=.003, d=0.86)$ and $21 \mathrm{~cm}(\mathrm{t}(17)=3.49, p=.003, d=0.83)$. The difference was approaching statistical significance for distractors presented at 42cm (t $(17)=2.86, p$ $=.011, d=0.61)$ and 42cm_opp (t $(17)=2.67, p=.016, d=0.60)$.

$<$ Figure 2 goes here $>$

$<$ Figure 3 goes here $>$

$A Q$ scores

Pearson's correlation coefficients (Bonferroni corrected $\alpha=.013$ ) were calculated to investigate the relationship between AQ scores and AASP scores (see Figure S2 in the supplementary materials). There was no significant correlation with sensory seeking items $(r=-.20, p=.431)$, low registration items $(r=.49, p=.037)$ or sensory sensitivity items $(r=.48, p=.043)$. There was a moderate to strong positive correlation with sensory avoidant items $(r=.67, p=.002)$.

Pearson's correlation coefficients (Bonferroni corrected $\alpha=.025$ ) were calculated to investigate the relationship between $A Q$ score and both tactile thresholds and CEs. AQ score did not correlate significantly with thresholds in Experiment $1(r=.38, p=.116)$ or Experiment $2(r=.26, p=.299)$, or with the size of the CE for any SOA, or position $(r<.33, p>.178$; see Figure S2 in the supplementary materials).

\section{Discussion}

The current study compared the temporal and spatial modulation of visual-tactile interactions between adults with high-functioning ASC and a matched NT control group. Evidence of altered sensory function was confirmed in participants with ASC using the AASP (Brown \& Dunn, 2002). Compared to NT participants, those with ASC rated low registration items significantly more highly and sensory seeking items significantly lower (see Table 1). There was no significant difference in tactile temporal separation thresholds between the groups. In the temporal task (Experiment 1), the congruency effect (CE) was significantly larger when stimuli were presented near simultaneously $(-30 \mathrm{~ms})$ than at all 
other stimulus onset asynchronies (SOA) for both groups. Comparisons with a unisensory baseline revealed evidence of multisensory interactions at $-30 \mathrm{~ms}$ and $100 \mathrm{~ms}$ for NT, but only $-30 \mathrm{~ms}$ for ASC participants. The NT group exhibited a significantly increased error rate to incongruent distractors presented $100 \mathrm{~ms}$ after the target in comparison to baseline trials, but this was absent in the group with ASC. In the spatial task (Experiment 2), the congruency effect was significantly larger for visual stimuli presented $0 \mathrm{~cm}$ and $21 \mathrm{~cm}$ compared to $42 \mathrm{~cm}$ from the participant's hand. The baseline analysis revealed evidence of multisensory interactions for participants with ASC for visual stimuli presented $0 \mathrm{~cm}$ and $21 \mathrm{~cm}$ from the participant's hand and at a position $42 \mathrm{~cm}$ from the participants hand in the opposite hemispace. NT participants produced this effect only for visual distractors positioned at $0 \mathrm{~cm}$ and $21 \mathrm{~cm}$.

The between-group differences in trait sensory processing as measured by the AASP is consistent with previous self-reports in high-functioning adults with ASC (Crane et al., 2009). Furthermore, there was a significant positive correlation between $A Q$ score and sensory avoidance in the control group. This is in line with recent research which has suggested that autistic traits may be associated with altered sensory functioning in the NT population (Horder, Wilson, Mendez, \& Murphy, 2014; Robertson \& Simmons, 2013). It is important to characterise NT participants used as a control group since individual differences in sensory and autistic traits may potentially mask between-group differences on behavioural measures. However, it should be noted that there were no significant correlations between AQ scores and tactile thresholds (although see Poole et al., 2015), or the size of the CE at each SOA and position. This suggests that the null effect of group on the temporal modulation of visual-tactile interactions in Experiment 1 was not driven by the NT participants who reported above average levels of autistic traits.

Tactile stimuli were presented at threshold in an attempt to equate the task difficulty between, and within, the participant groups (Poole et al., 2015). To determine tactile thresholds we utilised a nonspatial task which relied on participant's ability to separate vibrotactile stimuli in time and these thresholds did not differ statistically between the groups. Although it was not an explicit aim of the current study, this null difference contributes to the literature on the temporal processing of tactile stimuli in ASC. A previous study employed two tasks in which participants made simultaneity judgements and temporal order judgements regarding vibrotactile stimuli presented to different 
locations on the participant's hands (Tommerdahl, Tannan, Holden, \& Baranek, 2008). Adults with ASC produced higher thresholds than NT controls on both tasks when stimuli were delivered to the same hand, suggesting that longer durations were required to separate the vibrations (although see Puts et al., 2014, for different findings in children). When stimuli were presented to different hands, performance was comparable to controls. It may be that the ability to separate vibrotactile stimuli in time is intact in adults with ASC, but issues arise when separating stimuli that occur at different positions on the same limb. The issue of how time and space interact when individuals with ASC process tactile stimuli merits further investigation.

Typical audiovisual temporal modulation has previously been reported in adolescents and young adults with ASC (de Boer-Schellekens et al., 2013) and the current study extends this to the visualtactile modalities. Both groups produced a typical temporal profile of visual-tactile interactions previously established using the CCT (Shore et al., 2006). Interestingly, the findings from the baseline analysis suggest, that if anything, temporal modulation was stronger in participants with ASC. These findings contrast with reports of multisensory interactions in children with ASC over a range of SOAs double in size to NT children (Foss-Feig et al., 2010; Kwakye et al., 2011). The temporal modulation of multisensory interactions appears to be a complex developmental process, maturing across childhood and into late adolescence in the NT population (Hillock-Dunn \& Wallace, 2012). Therefore, a developmental delay in the temporal modulation of multisensory interactions in ASC may account for these apparently contradictory findings (see also, Stevenson, Siemann, Woynaroski et al., 2014). Although it is also plausible that compensatory strategies, for instance top-down attentional mechanisms (Talsma, Senkowski, Soto-Faraco, \& Woldorff, 2010), may have been established by adulthood. Top-down selection may 'step-in' to regulate how multisensory stimuli are processed when separated in time, perhaps driven by the strength of association between pairs of stimuli. Further work is required to confirm if, and when, temporal modulation stabilises in ASC.

We also investigated spatial modulation of multisensory interactions in ASC for the first time and provide preliminary evidence that this may be affected ${ }^{2}$. Both groups made significantly more errors in comparison to baseline when incongruent distractors were presented next to or $21 \mathrm{cms}$ away from

\footnotetext{
${ }^{2}$ A post-hoc power analysis on the trend towards an interaction for Group x Position suggests a sample of 20 participants in each group would be required to find a statistically significant effect where $\beta=0.84$.
} 
the stimulated hand. Participants with ASC also showed this effect when distractors were presented in the opposite hemispace (the $42 \mathrm{~cm} \_o p p$ position). However, there was considerable variability in performance in Experiment 2. Both groups of participants made more errors in response to the distractors in Experiment 2 than Experiment 1, and between-participant variability was much larger. Thus, selectively attending to touch in the presence of visual distractors presented at multiple, unpredictable locations (Experiment 2) appears to be more demanding than when distractors are presented in a predictable location, at different time points (Experiment 1). These task demands may have disproportionately affected the participants with ASC and thus the measurement of spatial modulation in this population, so it will be important to replicate this finding using a different task. Nevertheless, we consider explanations for this potential alteration in spatial modulation below.

The current findings could reflect a specific difficulty in the spatial processing of cross-modal stimuli. The spatial modulation of visual-tactile interactions has previously shown to be affected in healthy ageing (Poliakoff, Ashworth, Lowe, \& Spence, 2006). Older participants, aged over 76, produced statistically comparable CEs to distractors presented at the opposite and stimulated hand. Individuals with ASC may have similar issues appropriately modulating visual-tactile interactions. This might imply that the representation of peripersonal space around the hand is extended into the contralateral hemispace in participants with ASC. Where responses to extraneous stimuli are not effectively modulated or inhibited, perceptual estimates are likely to be noisy. Increased noise is believed to contribute to sensory symptomology in ASC (Pellicano, 2013), for instance, vision may have an atypical effect on the experience, or expectation of being touched. Our findings also fit with the recent observation that tactile judgements are less affected in participants with ASC when the arms are crossed over the body midline (Wada et al., 2014). This suggests that tactile spatial representations may be less affected by a visual frame-of-reference in ASC.

It is also important to consider a more general attentional account for the influence of more distant distractors in the ASC group. One possibility is that this finding reflects a difficulty in selectively attending to one location that can also be observed purely within the visual modality (Burack, 1994). Alternatively, participants with ASC may process the more distant distractor as they have a higher perceptual capacity (Remington, Swettenham, \& Lavie, 2012; Remington, Swettenham, Campbell, \& Coleman, 2009). Identifying the mechanisms which produce the increased processing of distant 
distractors will help improve our understanding of sensory processing in ASC. Therefore, future research should examine whether similar effects are observed within and across sensory modalities, exploring different attentional mechanisms such as spatial attention, multisensory selective attention and perceptual load.

In summary, the present study has revealed intact temporal modulation of visual-tactile interactions in a group of adults with ASC with self-reported sensory symptoms. This extends the characterisation of the temporal aspects of multisensory interactions in ASC to visual-tactile interactions in an adult sample. We also provide evidence that the spatial modulation of these interactions may be altered in ASC. Further studies are needed to replicate this effect using different tasks and to explore whether this reflects a more general attentional effect or a specific change in visual-tactile processing, which may relate to how people with ASC represent the space around their body.

\section{References}

American Psychiatric Association. (2013). Diagnostic and statistical manual of mental disorders (5th ed.). Arlington,VA: American Psychiatric Publishing.

Anderson, J. S., Druzgal, T. J., Froehlich, A., DuBray, M. B., Lange, N., Alexander, A. L., Abildskov, T., Nielsen, J.A., Cariello, A.N, Cooperrider, J.R., Bigler, E.D. \& Lainhart, J. E. (2011). Decreased interhemispheric functional connectivity in autism. Cerebral Cortex, 21(5), 11341146. doi:10.1093/cercor/bhq190

Baron-Cohen, S., Wheelwright, S., Skinner, R., Martin, J., \& Clubley, E. (2001). The autism-spectrum quotient (AQ): evidence from Asperger syndrome/high-functioning autism, males and females, scientists and mathematicians. Journal of Autism and Developmental Disorders, 31(1), 5-17.

Blakemore, S.-J., Tavassoli, T., Calò, S., Thomas, R. M., Catmur, C., Frith, U., \& Haggard, P. (2006). Tactile sensitivity in Asperger syndrome. Brain and Cognition, 61(1), 5-13. doi:10.1016/j.bandc.2005.12.013

Brandwein, A. B., Foxe, J. J., Butler, J. S., Russo, N. N., Altschuler, T. S., Gomes, H., \& Molholm, S. (2013). The development of multisensory integration in high-functioning autism: high-density electrical mapping and psychophysical measures reveal impairments in the processing of audiovisual inputs. Cerebral Cortex, 23(6), 1329-1341. doi:10.1093/cercor/bhs109

Brock, J., Brown, C. C., Boucher, J., \& Rippon, G. (2002). The temporal binding deficit hypothesis of autism. Development and Psychopathology, 14(2), 209-224.

Brown, C., \& Dunn, W. (2002). Adolescent/ Adult Sensory Profile. The Psychological Corporation, Texas, USA.

Burack, J. A. (1994). Selective attention deficits in persons with autism: preliminary evidence of an inefficient attentional lens. Journal of Abnormal Psychology, 103(3), 535-543. 
Collignon, O., Charbonneau, G., Peters, F., Nassim, M., Lassonde, M., Lepore, F., Mottron, L. \& Bertone, A. (2013). Reduced multisensory facilitation in persons with autism. Cortex, 49(6), 1704-1710. doi:10.1016/j.cortex.2012.06.001

Cousineau, D. (2005). Confidence intervals in within-subjects designs: a simpler solution to Loftus and Masson's method. Tutorials in Quantitative Methods for Psychology, 1(1), 42-45.

Crane, L., Goddard, L., \& Pring, L. (2009). Sensory processing in adults with autism spectrum disorders. Autism, 13(3), 215-228. doi:10.1177/1362361309103794

De Boer-Schellekens, L., Keetels, M., Eussen, M., \& Vroomen, J. (2013). No evidence for impaired multisensory integration of low-level audiovisual stimuli in adolescents and young adults with autism spectrum disorders. Neuropsychologia, 51(14), 3004-3013. doi:10.1016/j.neuropsychologia.2013.10.005

Foss-Feig, J. H., Kwakye, L. D., Cascio, C. J., Burnette, C. P., Kadivar, H., Stone, W. L., \& Wallace, M. T. (2010). An extended multisensory temporal binding window in autism spectrum disorders. Experimental Brain Research., 203(2), 381-389. doi:10.1007/s00221-010-2240-4

Geurts, H. M., Grasman, R. P. P. P., Verté, S., Oosterlaan, J., Roeyers, H., van Kammen, S. M., \& Sergeant, J. A. (2008). Intra-individual variability in ADHD, autism spectrum disorders and Tourette's syndrome. Neuropsychologia, 46(13), 3030-3041. doi:10.1016/j.neuropsychologia.2008.06.013

Gori, M., Del Viva, M., Sandini, G., \& Burr, D. C. (2008). Young children do not integrate visual and haptic form information. Current Biology, 18(9), 694-8. doi:10.1016/j.cub.2008.04.036

Gowen, E., \& Hamilton, A. (2013). Motor abilities in autism: a review using a computational context. Journal of Autism and Developmental Disorders, 43(2), 323-344. doi:10.1007/s10803-012$1574-0$

Hazen, E. P., Stornelli, J. L., O'Rourke, J. A., Koesterer, K., \& McDougle, C. J. (2014). Sensory symptoms in autism spectrum disorders. Harvard Review of Psychiatry, 22(2), 112-124. doi:10.1097/01.HRP.0000445143.08773.58

Hillock-Dunn, A., \& Wallace, M. T. (2012). Developmental changes in the multisensory temporal binding window persist into adolescence. Developmental Science, 15(5), 688-696. doi:10.1111/j.1467-7687.2012.01171.x

Holmes, N. P., Sanabria, D., Calvert, G. a, \& Spence, C. (2006). Multisensory interactions follow the hands across the midline: evidence from a non-spatial visual-tactile congruency task. Brain Research, 1077(1), 108-115. doi:10.1016/j.brainres.2005.11.010

Holmes, N. P., Sanabria, D., Calvert, G. a, \& Spence, C. (2007). Tool-use: capturing multisensory spatial attention or extending multisensory peripersonal space? Cortex, 43(3), 469-489.

Horder, J., Wilson, C. E., Mendez, M. A., \& Murphy, D. G. (2014). Autistic traits and abnormal sensory experiences in adults. Journal of Autism and Developmental Disorders, 44(6), 1461-1469. doi:10.1007/s10803-013-2012-7

larocci, G., \& McDonald, J. (2006). Sensory integration and the perceptual experience of persons with autism. Journal of Autism and Developmental Disorders, 36(1), 77-90. doi:10.1007/s10803-005$0044-3$

larocci, G., Rombough, A., Yager, J., Weeks, D. J., \& Chua, R. (2010). Visual influences on speech perception in children with autism. Autism, 14(4), 305-320. doi:10.1177/1362361309353615 
Keane, B. P., Rosenthal, O., Chun, N. H., \& Shams, L. (2010). Audiovisual integration in high functioning adults with autism. Research in Autism Spectrum Disorders, 4(2), 276-289. doi:10.1016/j.rasd.2009.09.015

Kern, J. K., Trivedi, M. H., Garver, C. R., Grannemann, B. D., Andrews, A. a, Savla, J. S., Johnson, D. G., Mehta, J.A. \& Schroeder, J. L. (2006). The pattern of sensory processing abnormalities in autism. Autism, 10(5), 480-494. doi:10.1177/1362361306066564

Kikuchi, M., Yoshimura, Y., Hiraishi, H., Munesue, T., Hashimoto, T., Tsubokawa, T., Takahoshi, T., Suzuki, M., Higashida, H. \& Minabe, Y. (2014). Reduced long-range functional connectivity in young children with autism spectrum disorder. Social Cognitive and Affective Neuroscience, 10(2), 248-254. doi:10.1093/scan/nsu049

Kwakye, L. D., Foss-Feig, J. H., Cascio, C. J., Stone, W. L., \& Wallace, M. T. (2011). Altered auditory and multisensory temporal processing in autism spectrum disorders. Frontiers in Integrative Neuroscience, 4, 1-11. doi:10.3389/fnint.2010.00129

Leekam, S. R., Nieto, C., Libby, S. J., Wing, L., \& Gould, J. (2007). Describing the sensory abnormalities of children and adults with autism. Journal of Autism and Developmental Disorders, 37(5), 894-910. doi:10.1007/s10803-006-0218-7

Lloyd, D. M. (2009). The space between us: a neurophilosophical framework for the investigation of human interpersonal space. Neuroscience and Biobehavioral Reviews, 33(3), 297-304. doi:10.1016/j.neubiorev.2008.09.007

Lord, C., Rutter, M., DiLavore, P. C., \& Risi, S. (2012). Autism Diagnostic Observation Schedule, second edition (ADOS-2). Torrance, CA: Western Psychological Services.

MacDonald, J., \& McGurk, H. (1978). Visual influences on speech perception processes. Perception \& Psychophysics, 24(3), 253-257.

O'Neill, M., \& Jones, R. S. (1997). Sensory-perceptual abnormalities in autism: a case for more research? Journal of Autism and Developmental Disorders, 27(3), 283-293.

Oldfield, R. C. (1971). The assessment and analysis of handedness: the Edinburgh inventory. Neuropsychologia, 9(1), 97-113.

Pellicano, E. (2013). Sensory symptoms in autism: a blooming, buzzing confusion? Child Development Perspectives, 7(3), 143-148. doi:10.1111/cdep.12031

Poliakoff, E., Ashworth, S., Lowe, C., \& Spence, C. (2006). Vision and touch in ageing: crossmodal selective attention and visuotactile spatial interactions. Neuropsychologia, 44(4), 507-517. doi:10.1016/j.neuropsychologia.2005.07.004

Poole, D., Couth, S., Gowen, E., Warren, P. A., \& Poliakoff, E. (2015). Adapting the crossmodal congruency task for measuring the limits of visual-tactile interactions within and between groups. Multisensory Research. doi: 10.1163/22134808-00002475

Puts, N. A., Wodka, E. L., Tommerdahl, M., Mostofsky, S. H., \& Edden, R. A. (2014). Impaired tactile processing in children with Autism Spectrum Disorder. Journal of Neurophysiology, 1803-1811. doi:10.1152/jn.00890.2013

Remington, A. M., Swettenham, J. G., \& Lavie, N. (2012). Lightening the load: perceptual load impairs visual detection in typical adults but not in autism. Journal of Abnormal Psychology, 121(2), 544-551. doi:10.1037/a0027670 
Remington, A., Swettenham, J., Campbell, R., \& Coleman, M. (2009). Selective attention and perceptual load in autism spectrum disorder. Psychological Science, 20(11), 1388-1393. doi:10.1111/j.1467-9280.2009.02454.x

Robertson, A. E., \& Simmons, D. R. (2013). The relationship between sensory sensitivity and autistic traits in the general population. Journal of Autism and Developmental Disorders, 43(4), 775784. doi:10.1007/s10803-012-1608-7

Shams, L, Kamitani, Y., \& Shimojo, S. (2000). What you see is what you hear. Nature, 408, 788.

Shams, Ladan, Kamitani, Y., \& Shimojo, S. (2002). Visual illusion induced by sound. Brain Research. Cognitive Brain Research, 14(1), 147-152.

Shore, D. I., Barnes, M. E., \& Spence, C. (2006). Temporal aspects of the visuotactile congruency effect. Neuroscience Letters, 392(1-2), 96-100. doi:10.1016/j.neulet.2005.09.001

Smith, E. G., \& Bennetto, L. (2007). Audiovisual speech integration and lipreading in autism. Journal of Child Psychology and Psychiatry, 48(8), 813-821. doi:10.1111/j.1469-7610.2007.01766.x

Spence, C, Pavani, F., \& Driver, J. (1998). What crossing the hands can reveal about crossmodal links in spatial attention. Abstracts of the Psychonomic Society, $3,13$.

Spence, Charles, Pavani, F., \& Driver, J. (2004). Spatial constraints on visual-tactile cross-modal distractor congruency effects. Cognitive, affective \& behavioral neuroscience, 4(2), 148-169.

Spence, Charles, Pavani, F., Maravita, A., \& Holmes, N. (2004). Multisensory contributions to the 3-D representation of visuotactile peripersonal space in humans: evidence from the crossmodal congruency task. Journal of Physiology, 98(1-3), 171-189. doi:10.1016/j.jphysparis.2004.03.008

Stevenson, R. a, Siemann, J. K., Woynaroski, T. G., Schneider, B. C., Eberly, H. E., Camarata, S. M., \& Wallace, M. T. (2014). Evidence for Diminished Multisensory Integration in Autism Spectrum Disorders. Journal of Autism and Developmental Disorders. doi:10.1007/s10803-014-2179-6

Stevenson, R. A., Siemann, J. K., Schneider, B. C., Eberly, H. E., Woynaroski, T. G., Camarata, S. M., \& Wallace, M. T. (2014). Multisensory temporal integration in autism spectrum disorders. The Journal of Neuroscience, 34(3), 691-697. doi:10.1523/JNEUROSCI.3615-13.2014

Talsma, D., Senkowski, D., Soto-Faraco, S., \& Woldorff, M. G. (2010). The multifaceted interplay between attention and multisensory integration. Trends in Cognitive Sciences, 14(9), 400-410. doi:10.1016/j.tics.2010.06.008

Taylor, N., Isaac, C., \& Milne, E. (2010). A comparison of the development of audiovisual integration in children with autism spectrum disorders and typically developing children. Journal of Autism and Developmental Disorders, 40(11), 1403-1411. doi:10.1007/s10803-010-1000-4

Taylor, M, Creelman, C. D. (1967). PEST : Efficient Estimates on Probability Functions. Acoustical Society of America, 41(4), 4-9.

Tommerdahl, M., Tannan, V., Holden, J. K., \& Baranek, G. T. (2008). Absence of stimulus-driven synchronization effects on sensory perception in autism: Evidence for local underconnectivity? Behavioral and Brain Functions, 4, 19. doi:10.1186/1744-9081-4-19

Van der Smagt, M. J., van Engeland, H., \& Kemner, C. (2007). Can you see what is not there? Lowlevel auditory-visual integration in autism spectrum disorder. Journal of Autism and Developmental Disorders, 37(10), 2014-2019. doi:10.1007/s10803-006-0346-0 
Wada, M., Suzuki, M., Takaki, A., Miyao, M., Spence, C., \& Kansaku, K. (2014). Spatio-temporal processing of tactile stimuli in autistic children. Scientific Reports, 4, 5985. doi:10.1038/srep05985

Weschler, D. (1999). Weschler Abbreviated Scale of Intelligence. San Antonia: The Psychological Corporation, Texas, USA.

Williams, J. H. G., Massaro, D. W., Peel, N. J., Bosseler, A., \& Suddendorf, T. (2004). Visual-auditory integration during speech imitation in autism. Research in Developmental Disabilities, 25(6), 559-575. doi:10.1016/j.ridd.2004.01.008 
Figure Caption Sheet

Figure 1 Schematic of the experimental set-up including the position of each LED for a right handed participant. The positions of the LEDs were equivalent for left handed participants. Participants were instructed to keep their non-dominant hand underneath the desk throughout the testing session. The photograph displays the bone conductor, foam cube and the LED distractor in the $0 \mathrm{~cm}$ position.

Figure 2 Congruency effect (\% error) at each SOA (Experiment 1, A) and position (Experiment 2, B). The congruency effect is calculated by deducting the error rate in incongruent trials from congruent trials for each group at each position. ASC participants are represented by dark grey bars, NT participants are represented by lighter grey. Error bars represent standard error of the condition means

Figure 3 Mean error rate for congruent (diamonds) and incongruent trials (squares) at each SOA (Experiment 1, A), and position (Experiment 2, B) for ASC and NT participants. Mean error rate in the unisensory baseline condition is represented for each group in each experiment by the dotted line. Asterisks denote a significant difference from baseline (Bonferroni corrected, $\alpha=.006$ ). Error bars denote within participants confidence intervals (Cousineau, 2005) 
22

Figure 1 top

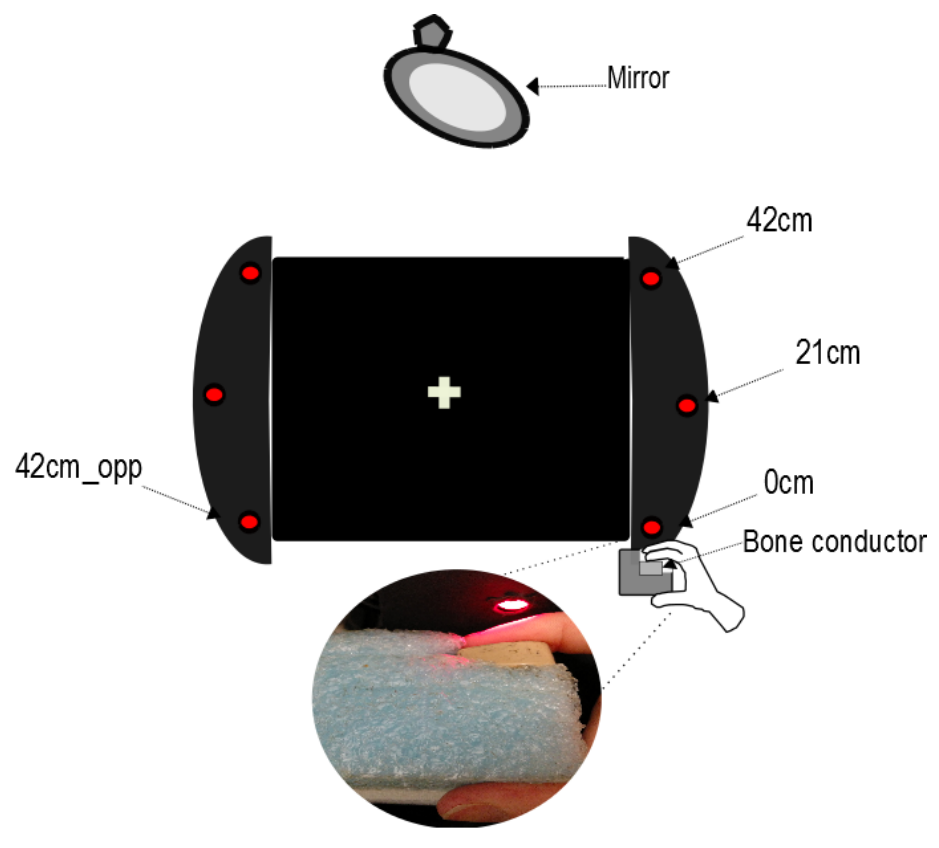


Figure 2 top
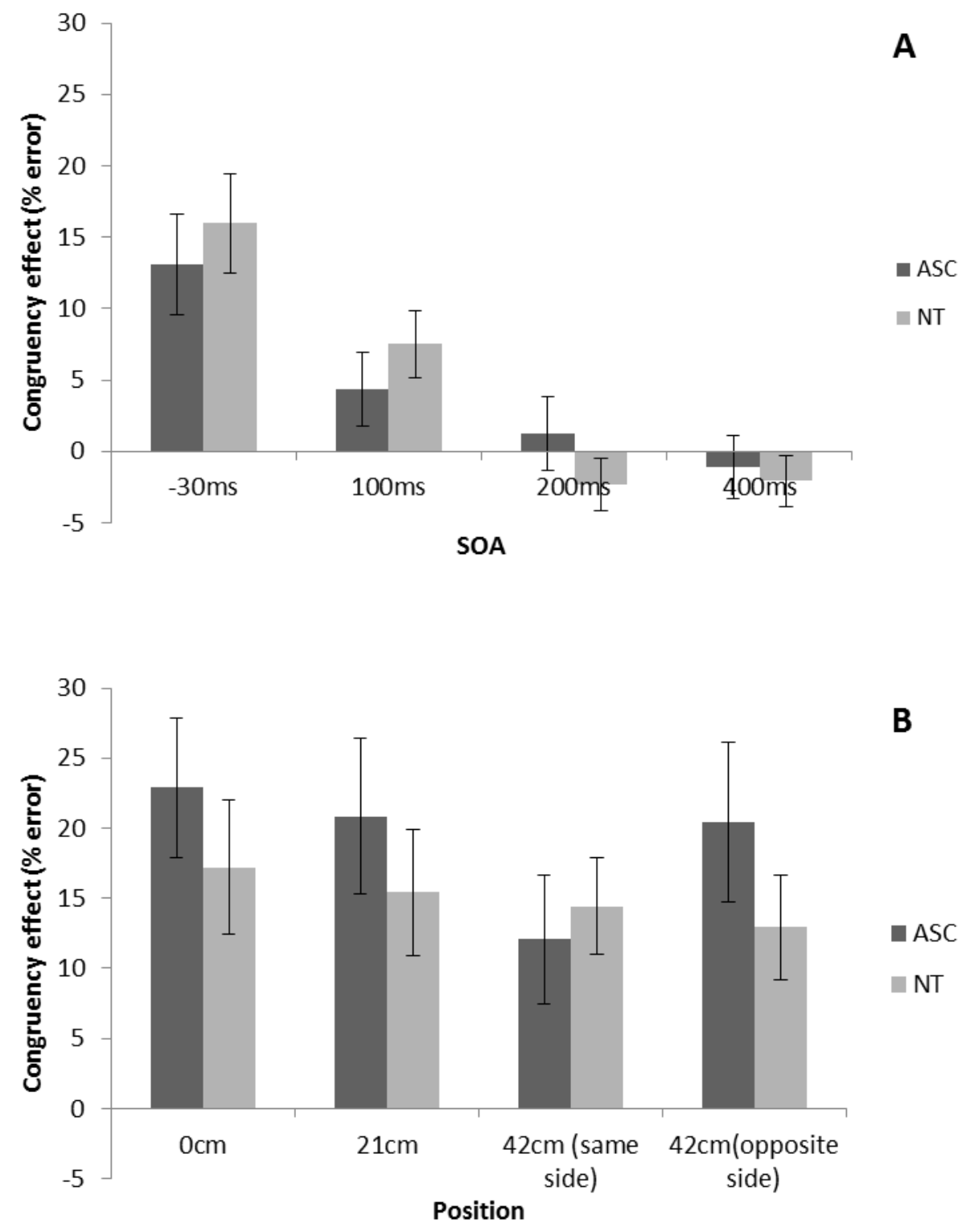
Figure 3 Top
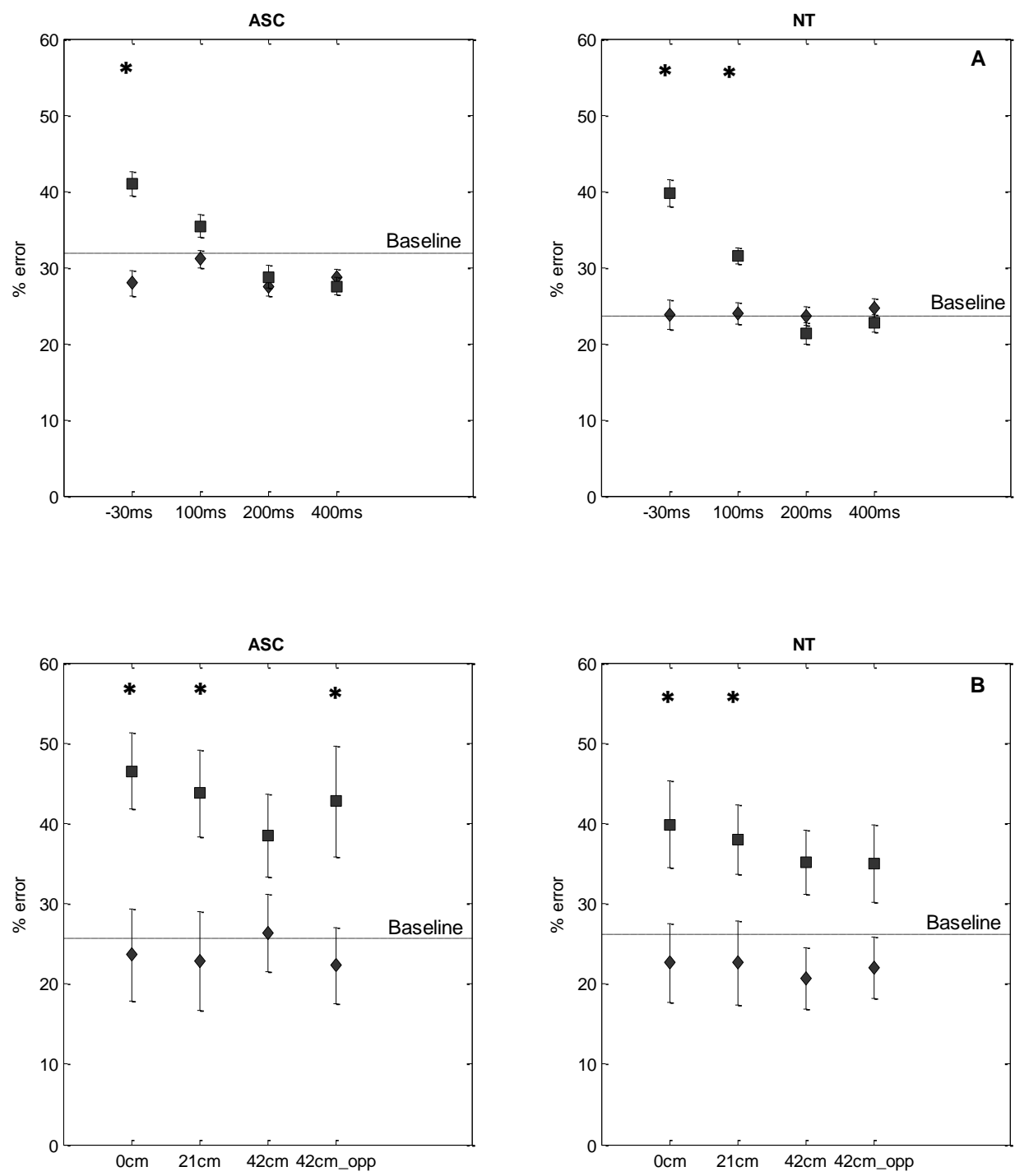
Table 1 Characteristics of participants with ASC and NT, including t-test statistics. Mean \pm standard deviations values are presented.

\begin{tabular}{|c|c|c|c|c|c|}
\hline & & ASC & NT & $t(34)$ & $p$ \\
\hline & Age & $29.8 \pm 8.1$ & $29.1 \pm 7.2$ & .23 & .819 \\
\hline & FSIQ & $118.3 \pm 9.9$ & $117.6 \pm 13.4$ & .05 & .960 \\
\hline & ASQ & - & $17.61 \pm 5.63$ & - & - \\
\hline & ADOS & $9.00 \pm 1.75$ & - & - & - \\
\hline AASP Quadrant & Sensory seeking & $37.11 \pm 6.27$ & $45.22 \pm 7.54$ & 3.56 & $.001 *$ \\
\hline Score & Low registration & $44.89 \pm 8.15$ & $34.67 \pm 7.26$ & 3.97 & $<.001 *$ \\
\hline (Bonferroni & Sensory avoidance & $45.17 \pm 9.95$ & $37.78 \pm 8.09$ & 2.44 & .020 \\
\hline corrected, $\alpha=.013$ ) & Sensory sensitivity & $42.56 \pm 7.65$ & $37.06 \pm 5.78$ & 2.44 & .020 \\
\hline Tactile Temporal & Experiment 1 & $25.82 \pm 12.67$ & $26.06 \pm 5.82$ & 0.70 & .944 \\
\hline \multicolumn{6}{|l|}{ Separation } \\
\hline Threshold (ms) & Experiment 2 & $28.24 \pm 11.73$ & $26.50 \pm 7.21$ & (33).53 & .599 \\
\hline
\end{tabular}

